Meta

Journal des traducteurs

Translators' Journal

\title{
Les fonctions du service de traduction
}

\section{Gilles Colpron}

Volume 21, numéro 1, mars 1976

La traduction et l'entreprise

URI : https://id.erudit.org/iderudit/002352ar

DOI : https://doi.org/10.7202/002352ar

Aller au sommaire du numéro

Éditeur(s)

Les Presses de l'Université de Montréal

ISSN

0026-0452 (imprimé)

1492-1421 (numérique)

Découvrir la revue

Citer cet article

Colpron, G. (1976). Les fonctions du service de traduction. Meta, 21(1), 64-67.

https://doi.org/10.7202/002352ar d'utilisation que vous pouvez consulter en ligne.

https://apropos.erudit.org/fr/usagers/politique-dutilisation/ 


\section{Les fonctions du service de traduction}

Le service de traduction, dans une entreprise du type québécois courant, devient en fait le «service linguistique » ou «les services linguistiques», du fait que l'appellation de service de traduction n'est pas assez vaste, le plus souvent, pour recouvrir toutes ses activités. Effectivement, on rencontre dans les entreprises de ce genre, à côté de «Bureau de traduction» et «Centre de traduction», les noms de "Services linguistiques», «Service de linguistique», "Service traduction et linguistique », "Service traduction et terminologie», dans un effort, semble-t-il, pour indiquer que les fonctions du service embrassent un certain nombre d'activités annexes à la traduction. Nous laissons de côté le nom de «Services français» comme ne représentant pas un ensemble de fonctions à caractère proprement linguistique mais désignant plutôt le pendant français de différents services pour le public, pendant qui englobe des agents de relations publiques, des publicitaires, des représentants divers (bien que dans la pratique, l'appellation soit souvent fausse, les membres des French Services ou du French Group étant confinés dans un rôle de traducteurs). Il est entendu que nous ne traitons pas ici des entreprises purement commerciales, des entreprises de genre particulier comme les agences de publicité, ni des toutes petites entreprises. Par « entreprise du type québécois courant », nous entendons une société qui produit et vend des biens ou des services (souvent les deux à la fois), cont la haute direction et les cadres sont largement anglophones et qui compte donc une minorité plus ou moins importante de francophones dans son personnel de gestion. Notre description sera basée sur les entreprises de grande taille, mais on est fondé à penser que la situation ne diffère pas beaucoup chez les entreprises de taille moyenne.

Le service de traduction (gardons cette expression en guise de dénominateur commun, d'autant plus qu'elle correspond à l'activité principale du service - que nous appellerons commodément aussi, ci-après, le Service) est chargé de la traduction de tous les genres de documents que l'entreprise peut produire. Seuls lui échappent dans certains cas, en partie ou en totalité, les annonces commerciales, les communiqués pour les journaux, le périodique interne de l'entreprise et parfois la revue externe, la correspondance courante et des textes courts, comme des affiches, des pancartes, des panneaux indicateurs, des cartes de visite. Le pourcentage des textes de ce genre qui n'est pas confié au service de traduction dépend de la présence plus ou moins importante de francophones au service de la publicité 
et des relations publiques (ou encore au service des publications et à celui du personnel), de secrétaires ou d'autres employés réputés capables de traduire les lettres dans les différents services administratifs, et enfin, de cadres et d'employés de langue française dans les usines, entrepôts et établissements assimilés de la société. En ce qui concerne ce dernier genre d'établissement, l'importance que l'on accorde à l'affiche et l'empire qu'a su ou n'a pas su établir le service de traduction, posté au siège social, entrent aussi en ligne de compte.

Le service traduit une gamme si vaste de textes qu'on sent le besoin de les regrouper en grandes catégories plutôt que d'en faire une énumération complète, qui serait d'ailleurs difficilement exhaustive. On peut distinguer: 1) les documents pour le public: prospectus, brochures et bulletins à caractère publicitaire, catalogues, guides d'utilisation, articles pour le périodique externe;2) les documents pour des publics restreints : rapports aux actionnaires, notices financières, mémoires au gouvernement ou à des organismes officiels, discours, textes de films, notices techniques; 3) les textes pour des particuliers: documents à caractère commercial et juridique (contrats, baux, soumissions, devis), lettres importantes ; 4) les textes pour les salariés : circulaires, articles pour le journal d'entreprise, réglementations, manuels de méthodes, formules, régimes d'avantages sociaux, etc.

Le «sens » de la traduction est de l'anglais au français, généralement dans une très forte proportion. Pour ce qui est des entreprises ayant leur siège dans la région montréalaise (nous manquons de renseignements sur les autres, peu nombreuses, d'ailleurs, à être dotées d'un service de traduction), la proportion de la traduction du français à l'anglais va de négligeable à près de 35 pour cent. Contrairement à ce qu'on aurait pu attendre, les pourcentages les plus élevés, en cette matière, n'indiquent pas une plus forte présence de francophones investis de responsabilités, mais peut-être un caractère plus anglophone unilingue: il s'agit d'entreprises qui se font traduire une certaine documentation française publiée par le gouvernement du Québec, certains articles de journaux et surtout des documents venant d'Europe et d'Afrique francophone, où elles font des affaires. Dans un certain nombre d'entreprises, il se fait aussi un peu de traduction en langues étrangères (principalement espagnol, allemand, japonais, suédois, portugais, italien, russe). Surtout lorsqu'elle va de l'anglais vers la langue étrangère, cette traduction est habituellement confiée par le service de traduction à des spécialistes de l'extérieur.

Le deuxième rôle du service de traduction est de réviser les textes traduits ou rédigés dans les autres services ou subdivisions. Pour les textes de peu d'importance ou de diffusion très restreinte, surtout à l'intérieur de l'entreprise, on ne s'oppose habituellement pas à ce que des gens extérieurs à la profession les traduisent : le service a mieux à faire que de traduire une masse de documents insignifiants quant à leur portée. Il est de plus favorable, habituellement, à ce que les employés ou les gestionnaires francophones écrivent directement en français, se disant que mieux vaut pour eux de s'exprimer plus ou moins bien que de ne pas s'exprimer du tout, et que si le français doit devenir la langue principale de travail, il leur faut apprendre à écrire en français. Le service insiste cependant pour qu'on lui soumette les textes français de quelque importance produits ailleurs que chez lui. De là vient qu'il est appelé à réviser des textes (venant surtout des usines, en général fortement francophones) et à donner des conseils d'ordre syntaxique, stylistique et terminolo- 
gique. Il fournit volontiers aussi des consultations de même ordre, surtout par téléphone, lorsqu'il estime ne pas devoir réviser le texte entier. Il lui arrive également d'être consulté sur des questions d'impression, de disposition, de présentation physique d'un texte (par exemple, d'une formule qui pourrait soit comporter les deux langues sur une même face et dans le même ensemble de cases, soit avoir l'autre langue au verso, soit être présentée sous forme de deux documents parallèles unilingues). On voit donc que le service de traduction ne travaille pas dans l'abstrait, mais veille à ce que les textes atteignent les lecteurs dans un état de correction concrète. C'est d'ailleurs la raison pour laquelle, à sa propre demande, il corrige les épreuves des documents diffusés sous forme imprimée.

Dépassant ce rôle de conseil, le service exerce, dans beaucoup de cas, une espèce de direction linguistique, bien que son autorité soit surtout morale, vu qu'il est un fournisseur de services et ne saurait forcer ses « clients » à employer, dans des documents publiés en leur nom, des termes et tournures dont ils ne veulent pas. Tout de même, ses attributions le définissent souvent comme l'autorité quant à la norme linguistique, et s'il possède l'art de la persuasion, qui suppose avoir su inspirer la confiance et le respect par des avis compétents, bien motivés et éclairés, il peut obtenir que ses décisions soient suivies et concourir au redressement de la langue courante dans l'ensemble de l'entreprise. Toutefois, il semble que des appuis en haut lieu, ou encore l'établissement officiel de son autorité, soient nécessaires pour changer certaines habitudes consacrées par toute la traditions écrite aussi bien qu'orale du domaine d'activité concerné : nous pensons ici en particulier aux relations syndicales-patronales et au secteur juridique (conventions collectives, régimes de sécurité sociale), où presque tous les documents reproduisant l'usage professionnel et même les lois attestant de l'usage de l'État sont effroyablement anglicisés et «créolisés ». C'est dire que l'État du Québec empêche les services linguistiques des entreprises d'exécuter sa consigne de francisation par sa législation dans une langue abâtardie, sur laquelle s'appuient le service juridique et celui des relations de travail pour maintenir la tradition. Néanmoins, dans les autres secteurs, où ne joue pas l'impératif de protéger à tout prix les intérêts de la société auprès des salariés et des instances judiciaires, et là où aucun intérêt commercial n'est en cause (ce qui exclut en principe, par exemple, la description des produits à l'intention du public), le service de traduction exerce généralement une action normalisatrice réelle. Il peut décider seul - ou tout au moins il aura son grand mot à y dire - des appellations françaises des postes et des services, ce qui est déjà un élément important de francisation de l'usage. Pour le reste, il applique dans le langage écrit une norme qui demeurera selon qu'elle passera ou non inaperçue des intéressés (francophones et anglophones qui se piquent de connaître le français), qu'elle contredira ou non des habitudes vénérées ou qu'elle touchera des gens butés ou ouverts. Malgré des obstacles pratiques et des échecs particuliers qu'on peut d'ailleurs espérer momentanés, il demeure que le Service est investi d'un rôle de normalisation et de surveillance de la qualité du français dans l'entreprise, fonction qui peut ajouter une dimension plus stimulante à son travail.

Quant à la recherche terminologique, elle est une activité accessoire à la traduction; on peut même dire qu'elle fait partie intégrante de la traduction spécialisée ou technique. Elle devient toutefois une activité autonome lorsqu'elle prend 
la forme du dépouillement systématique, par opposition à la simple recherche, dans les revues et autres documents français (ou anglais), de termes particuliers dont on a besoin sur le moment. Le dépouillement vise à constituer des réserves de terminologie en vue de prévenir les besoins des traducteurs et de constituer des vocabulaires complets qui pourront prendre la forme de lexiques, accessibles au personnel de l'entreprise, aux autres bureaux de traduction et même au grand public. L'activité recherche, surtout si elle comporte du dépouillement, commande généralement un outillage important (bibliothèque très spécialisée et fichier considérable, tous deux classés et référencés de façon très étudiée) et un personnel attitré (un ou des terminologues, un ou des documentalistes). Cette activité subsidiaire et complémentaire devient une véritable fonction du Service lorsque l'entreprise a donné son accord à la francisation de certains de ses établissements, opération qui consiste surtout à substituer les termes techniques anglais de la langue parlée des salariés francophones et à fournir à ceux-ci une documentation en français. Le Service est alors chargé de trouver le vocabulaire français que les techniciens et ouvriers emploieront au travail et qui figurera sur les formules, communications et documents divers servant dans les usines. Il traduira habituellement lui-même les documents permanents et sera appelé à établir des listes d'équivalents français en regard de termes anglais, devant permettre aux salariés de franciser leur langue parlée. Le Service, on le voit, est un instrument important dans l'exécution de la politique de francisation de l'entreprise et, pour peu que cette politique soit sérieuse, sinon commandée par un impératif vital, l'importance de son rôle s'en trouve accrue d'autant.

Certains services de traduction, débordant les fonctions que nous venons de décrire et devenant des services linguistiques au sens le plus large où on peut entendre l'expression, s'occupent aussi de la formation linguistique institutionnalisée : enseignement de la rédacttion, cours de langue aux anglophones. En effet, la politique linguistique de l'entreprise prévoit souvent comme principale mesure de francisation la « bilinguisation 》 de son personnel.

À supposer que la francisation de l'entreprise se fasse réellement (ce qui suppose non pas la présence d'un service-machine qui traduise tous les documents en français, ni la capacité des anglophones de répondre au public et aux ouvriers francophones en un quelconque français, mais la création en français par un personnel qui soit de cette langue et fasse vivre l'entreprise dans cette langue), on peut prévoir que le service de traduction deviendra presque exclusivement un service de conseil, de recherches terminologiques, de révision, de rédaction et de traduction vers l'anglais et d'autres langues.

Gilles Colpron 